\begin{tabular}{|c|l|}
\hline Title & Persistence of Brownian motion in a shear flow \\
\hline Author(s) & Takikawa, Y oshinori; Orihara, Hiroshi \\
\hline Citation & $\begin{array}{l}\text { Physical review e, 88(6), 062111-1-062111-5 } \\
\text { https://doi.org/10.1103/PhysRevE.88.062111 }\end{array}$ \\
\hline Issue Date & 2013-12-06 \\
\hline Doc URL & http://hdl.handle.net/2115/54624 \\
\hline Type & article \\
\hline File Information & Persistence of Brownian motion in a shear flow.pdf \\
\hline
\end{tabular}

Instructions for use 


\title{
Persistence of Brownian motion in a shear flow
}

\author{
Yoshinori Takikawa and Hiroshi Orihara* \\ Division of Applied Physics, Faculty of Engineering, Hokkaido University, Sapporo 060-8628, Japan
}

(Received 17 June 2013; published 6 December 2013)

\begin{abstract}
The persistence of a Brownian particle in a shear flow is investigated. The persistence probability $P(t)$, which is the probability that the particle does not return to its initial position up to time $t$, is known to obey a power law $P(t) \propto t^{-\theta}$. Since the displacement of a particle along the flow direction due to convection is much larger than that due to Brownian motion, we define an alternative displacement in which the convection effect is removed. We derive theoretically the two-time correlation function and the persistence exponent $\theta$ of this displacement. The exponent has different values at short and long times. The theoretical results are compared with experiment and a good agreement is found.
\end{abstract}

DOI: 10.1103/PhysRevE.88.062111

PACS number(s): 05.40.-a, 83.50.Ax

\section{INTRODUCTION}

Persistence probability $P(t)$ has attracted much attention over the past decade because it plays an important role as a dynamic probe in many stochastic processes, indicating how long the system retains a memory of its initial configuration. This probability is defined as the probability that the stochastic variable $x(t)$ does not return to the origin up to time $t$. In many cases, it decays according to a power law $P(t) \sim t^{-\theta}$, thus defining the nontrivial persistence exponent $\theta$. This exponent has been studied experimentally and theoretically for fluctuating interfaces [1-3], critical dynamics [4], granular media [5,6], disordered environments [7-9], and polymer dynamics [10].

In recent years, theoretical studies focusing on Brownian particles in composite media [9], a time-dependent potential [11], and a shear flow [12] have been undertaken by Chakraborty et al. because the Brownian motion of a particle is the simplest stochastic process. In the case of free Brownian motion, the exact value of the persistence exponent was found to be $\theta=1 / 2$ [13]. Chakraborty showed by theoretical calculation and simulation that the persistence probability for a Brownian particle subject to a shear flow decays in the flow direction as a power law and exhibits two distinct regimes of decay [12]. For short times, $\theta=1 / 2$, which is the same as the case of free Brownian motion, while for long times, $\theta=1 / 4$ as a result of the shear flow effect. These are exact results, but for most cases it is difficult to obtain an exact value for the exponent.

For the calculation of persistence exponents, some approximation methods have been developed $[14,15]$. In general, the processes under consideration are nonstationary ones, which can be mapped to Gaussian stationary processes. For example, in the case of a free Brownian particle, the displacement of the particle $x(t)$ is not a stationary variable. Therefore, the two-time correlation function $\left\langle x\left(t_{1}\right) x\left(t_{2}\right)\right\rangle$ is not a function of the difference of two times $t_{2}-t_{1}$, but, by defining a different variable $X(t) \equiv x(t) / \sqrt{\left\langle x^{2}(t)\right\rangle}$ and a different time variable $T=\ln t$, the two-time correlation function $\left\langle X\left(T_{1}\right) X\left(T_{2}\right)\right\rangle$ is a function of $\left|T_{2}-T_{1}\right|$, that is, the stochastic process $X(T)$ is stationary. It is known that the persistence probability for a

*orihara@eng.hokudai.ac.jp
Gaussian stationary process usually decays as $\sim \exp (-\theta T)$ for large $T=\left|T_{2}-T_{1}\right|$, and methods have been devised to calculate the persistence exponent $\theta$ from the two-time correlation function.

As far as the authors know, the persistence exponents for the sheared Brownian particle and the free one still need to be found experimentally. Recently, to obtain the mean-square displacement (MSD) of a particle in a simple shear flow, we have performed an experiment using confocal scanning laser microscopy. In a simple shear flow, the displacement of a particle in the flow direction includes convection, which makes a much larger contribution to MSD than the Brownian motion. Here, we consider a particle in a simple shear flow along the $x$ axis with the velocity gradient in the $z$ direction. The $x$ coordinate of the particle at time $t$ can be described as $x(t)=x(0)+z(0) \dot{\gamma} t+x_{B}(t)$, where $x(0)$ and $z(0)$ are the initial coordinates, $\dot{\gamma}$ is the shear rate, and $x_{B}(t)$ indicates the contribution from the Brownian motion, the MSD of which is given by $\left\langle x_{B}(t)^{2}\right\rangle=2 D t\left[1+(\dot{\gamma} t)^{2} / 3\right]$ with diffusion constant $D$. Note that $x_{B}(0)=0$, independent of the initial coordinates $x(0)$ and $z(0)$. In experiments, we can obtain $\left\langle[x(t)-x(0)]^{2}\right\rangle=[z(0) \dot{\gamma}]^{2} t^{2}+\left\langle x_{B}(t)^{2}\right\rangle$, which includes a large $t^{2}$ term, but it is quite difficult to measure $z(0)$ with sufficient accuracy to calculate $\left\langle[x(t)-x(0)-z(0) \dot{\gamma} t]^{2}\right\rangle=$ $\left\langle x_{B}(t)^{2}\right\rangle$. In order to overcome this difficulty, we introduced a variable [16],

$$
\tilde{x}(t) \equiv\{[x(2 t)-x(t)]-[x(t)-x(0)]\} / \sqrt{2} .
$$

As $[x(2 t)-x(t)]$ and $[x(t)-x(0)]$ include equal displacements due to convection during an interval of length $t$, the effects of convection are canceled out. It can also be shown that $\left\langle\tilde{x}(t)^{2}\right\rangle=\left\langle x_{B}(t)^{2}\right\rangle$.

In the next section, we calculate the two-time correlation function and persistence exponent of $\tilde{x}(t)$, which will be shown to be different from that of $x_{B}(t)$. The theoretical results are compared with the experimental ones, which are obtained without and with a shear flow, in Sec. III. The last section is devoted to conclusions.

\section{CALCULATION OF THE TWO-TIME CORRELATION FUNCTION AND PERSISTENCE EXPONENT}

For the Brownian motion of a particle subjected to a steady shear flow with shear rate $\dot{\gamma}$, where the flow velocity 

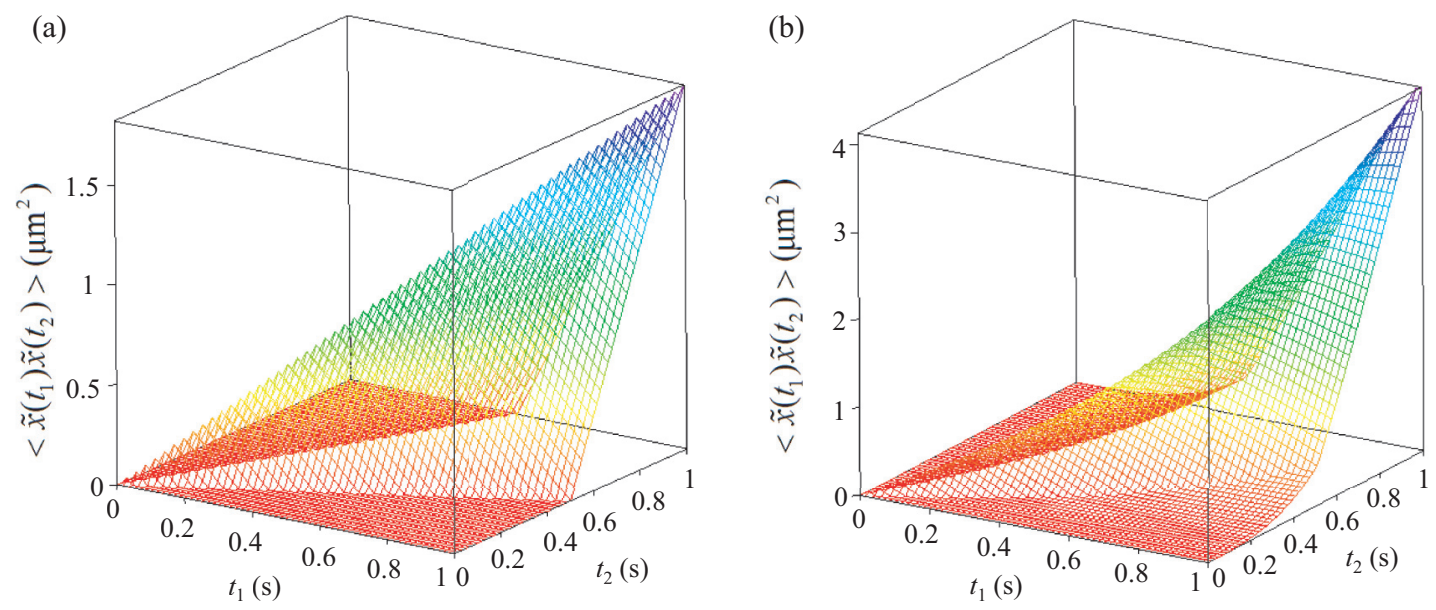

FIG. 1. (Color online) Three-dimensional (3D) plots of theoretically obtained two-time correlation functions of $\tilde{x}(t)$ for (a) $\dot{\gamma}=0$ and (b) $\dot{\gamma}=2 \mathrm{~s}^{-1}$.

and vorticity are in the $x$ and $z$ directions, respectively, the Langevin equation may be given by

$$
\begin{gathered}
\zeta[\dot{x}(t)-\dot{\gamma}(t) z(t)]=R_{x}(t), \\
\zeta z(t)=R_{z}(t),
\end{gathered}
$$

where $\zeta=6 \pi \eta a$, with $a$ being the particle radius and $\eta$ the shear viscosity. The random forces $R_{\alpha}(t)$ satisfy $\left\langle R_{\alpha}(t)\right\rangle=0$ and $\left\langle R_{\alpha}(t) R_{\beta}\left(t^{\prime}\right)\right\rangle=2 \zeta k_{B} T \delta_{\alpha \beta} \delta\left(t-t^{\prime}\right)$, where $k_{B}$ and $T$ are the Boltzmann constant and temperature, respectively. The inertia term has been dropped, as it is negligible for our experimental conditions. If we define $z_{B}(t)=z(t)-z(0)$, $x_{B}(t)$ and $z_{B}(t)$ satisfy Eq. (2) with initial conditions of $x_{B}(0)=z_{B}(0)=0$. The two-time correlation function of $x_{B}(t)$ under such conditions was obtained by Chakraborty [12]:

$$
\left\langle x_{B}\left(t_{1}\right) x_{B}\left(t_{2}\right)\right\rangle=2 D t_{2}+\dot{\gamma}^{2} D\left(t_{1} t_{2}^{2}-\frac{t_{2}^{3}}{3}\right) \text { for } t_{1} \geqslant t_{2},
$$

where $D=k_{B} T / \zeta$ is the diffusion constant. The two-time correlation function of $\tilde{x}(t)$ defined by Eq. (1) can be calculated from Eq. (3) as follows. From Eq. (1), we have

$$
\begin{aligned}
\left\langle\tilde{x}\left(t_{1}\right) \tilde{x}\left(t_{2}\right)\right\rangle= & \frac{1}{2}\left[\left\langle x_{B}\left(2 t_{1}\right) x_{B}\left(2 t_{2}\right)\right\rangle-2\left\langle x_{B}\left(2 t_{1}\right) x_{B}\left(t_{2}\right)\right\rangle\right. \\
& \left.-2\left\langle x_{B}\left(t_{1}\right) x_{B}\left(2 t_{2}\right)\right\rangle+4\left\langle x_{B}\left(t_{1}\right) x_{B}\left(t_{2}\right)\right\rangle\right] .
\end{aligned}
$$

It should be stressed that the two-time correlation function of $\tilde{x}(t)$ consists of that of $x_{B}(t)$, and is independent of the initial position. Substitution of Eq. (3) into Eq. (4) yields

$$
\begin{aligned}
& \left\langle\tilde{x}\left(t_{1}\right) \tilde{x}\left(t_{2}\right)\right\rangle \\
& =D\left\{4 t_{2}+\frac{1}{2} \dot{\gamma}^{2}\left(8 t_{1} t_{2}^{2}-\frac{10}{3} t_{2}^{3}\right)\right. \\
& \left.\quad-2\left[t_{1}+\frac{1}{2} \dot{\gamma}^{2}\left(2 t_{1}^{2} t_{2}-\frac{1}{3} t_{1}^{3}\right)\right]\right\} \quad\left(0 \leqslant t_{2} \leqslant t_{1} \leqslant 2 t_{2}\right),
\end{aligned}
$$

$$
\left\langle\tilde{x}\left(t_{1}\right) \tilde{x}\left(t_{2}\right)\right\rangle=D \dot{\gamma}^{2} t_{2}^{3} \quad\left(0<2 t_{2}<t_{1}\right) .
$$

The correlation function for $t_{1}<t_{2}$ is easily obtained using symmetry $\left\langle\tilde{x}\left(t_{1}\right) \tilde{x}\left(t_{2}\right)\right\rangle=\left\langle\tilde{x}\left(t_{2}\right) \tilde{x}\left(t_{1}\right)\right\rangle$. From Eqs. (3) and (5) it can be seen that $\left\langle\tilde{x}\left(t_{1}\right) \tilde{x}\left(t_{2}\right)\right\rangle$ is not identical to $\left\langle x\left(t_{1}\right) x\left(t_{2}\right)\right\rangle$ except for $t_{1}=t_{2}$, even without any shear flow. These facts indicate that stochastic properties, such as the persistence probability, should be different. The region $t_{2} \leqslant t_{1}$ is divided into two parts, $t_{1} \leqslant 2 t_{2}$ and $2 t_{2}<t_{1}$, in which the two-time correlations are given by Eqs. (5a) and (5b), respectively. The first derivatives of the two-time correlation function with respect to $t_{1}$ and $t_{2}$ at the boundary are discontinuous. When $2 t_{2}<t_{1}$, the two-time correlation function is zero without a shear flow, while in the presence of a shear flow it is finite. In order to visualize these features, the two-time correlation function for $\dot{\gamma}=0$ and $2 \mathrm{~s}^{-1}$ is shown as a function of $t_{1}$ and $t_{2}$ in Figs. 1(a) and 1(b), respectively, where we use the diffusion constant $D=0.94 \mu \mathrm{m}^{2} \mathrm{~s}^{-1}$ calculated from the Einstein-Stokes relation for the $0.5 \mu \mathrm{m}$ particle used in our experiment. For $\dot{\gamma}=0$ the boundaries $\left(2 t_{2}=t_{1}\right.$ and $\left.t_{2}=2 t_{1}\right)$ and the flat regions $\left(2 t_{2}<t_{1}\right.$ and $\left.t_{2}>2 t_{1}\right)$ are clearly seen [Fig. 1(a)]. For a finite shear rate [Fig. 1(b)], the flat regions change to curved surfaces as given by Eq. (5b), and the maximum value of the two-time correlation function at $t_{2}=t_{1}$ increases substantially for large $t_{1}$ due to the cubic terms in Eq. (5a).

In order to calculate the persistence exponent, we define a normalized variable $\tilde{X}(t)=\tilde{x}(t) / \sqrt{\left\langle\tilde{x}^{2}(t)\right\rangle}$. The two-time correlation function of $\tilde{X}(t)$,

$$
\left\langle\tilde{X}\left(t_{1}\right) \tilde{X}\left(t_{2}\right)\right\rangle=\frac{\left\langle\tilde{x}\left(t_{1}\right) \tilde{x}\left(t_{2}\right)\right\rangle}{\sqrt{\left\langle\tilde{x}\left(t_{1}\right)^{2}\right\rangle} \sqrt{\left\langle\tilde{x}\left(t_{2}\right)^{2}\right\rangle}},
$$

can be calculated from Eq. (5). In the short-time regime $(t \ll$ $\dot{\gamma}^{-1}$ ), the $t^{3}$ terms are negligible in Eq. (5), and, therefore, we get

$$
\left\langle\tilde{X}\left(t_{1}\right) \tilde{X}\left(t_{2}\right)\right\rangle=2\left(\frac{t_{2}}{t_{1}}\right)^{1 / 2}-\left(\frac{t_{1}}{t_{2}}\right)^{1 / 2} \quad\left(0 \leqslant t_{2} \leqslant t_{1} \leqslant 2 t_{2}\right),
$$

$$
\left\langle\tilde{X}\left(t_{1}\right) \tilde{X}\left(t_{2}\right)\right\rangle=0 \quad\left(0<2 t_{2}<t_{1}\right) .
$$




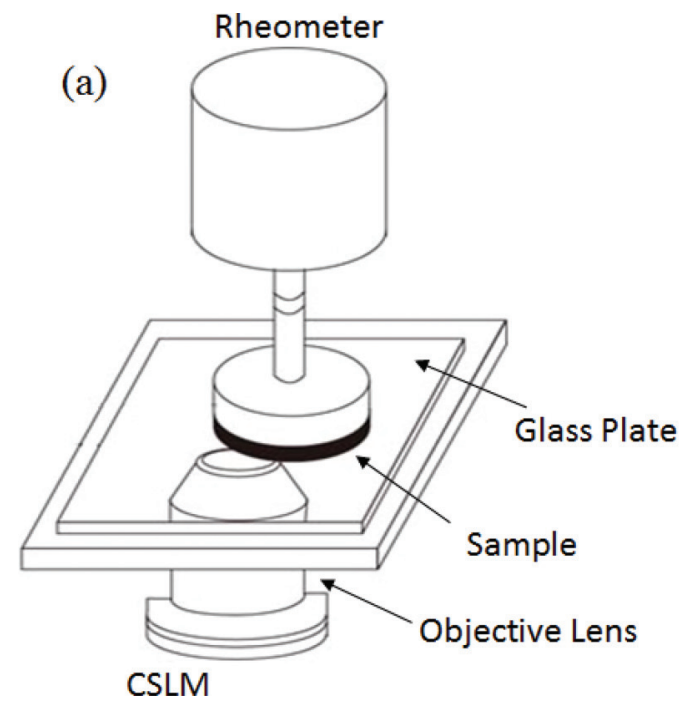

(b)

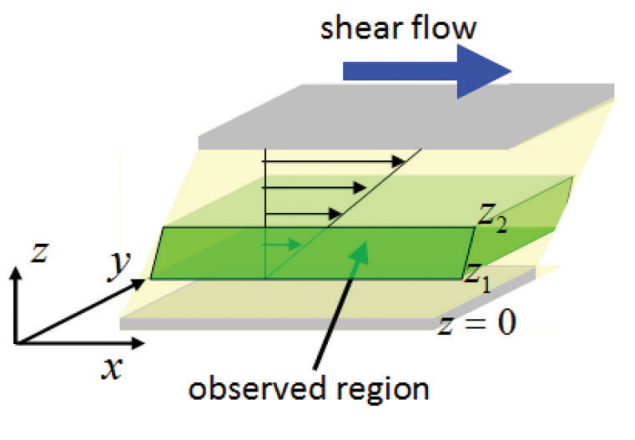

FIG. 2. (Color online) (a) Schematic illustration of our system combining a rheometer and CSLM. (b) Details of the sample space. The observed region is a thin layer between $z=z_{1}(5 \mu \mathrm{m})$ and $z=z_{2}(15 \mu \mathrm{m})$.

On the other hand, the long-time behavior $\left(t \gg \dot{\gamma}^{-1}\right)$ is given by neglecting the terms linear in $t$ in Eq. (5):

$$
\begin{aligned}
\left\langle\tilde{X}\left(t_{1}\right) \tilde{X}\left(t_{2}\right)\right\rangle= & 6\left(\frac{t_{2}}{t_{1}}\right)^{1 / 2}-\frac{5}{2}\left(\frac{t_{2}}{t_{1}}\right)^{3 / 2}-3\left(\frac{t_{1}}{t_{2}}\right)^{1 / 2} \\
& +\frac{1}{2}\left(\frac{t_{1}}{t_{2}}\right)^{3 / 2} \quad\left(0 \leqslant t_{2} \leqslant t_{1} \leqslant 2 t_{2}\right), \\
\left\langle\tilde{X}\left(t_{1}\right) \tilde{X}\left(t_{2}\right)\right\rangle= & \frac{3}{2}\left(\frac{t_{2}}{t_{1}}\right)^{3 / 2} \quad\left(0<2 t_{2}<t_{1}\right) .
\end{aligned}
$$

Introducing a different time variable $T=\ln t$, the stochastic process $\tilde{X}(T)$ becomes a Gaussian stationary process. The correlation function $C(T)=\langle\tilde{X}(T) \tilde{X}(0)\rangle$ for $t \ll \dot{\gamma}^{-1}$ is

$$
\begin{gathered}
C(T)=2 e^{-T / 2}-e^{T / 2}(0 \leqslant T \leqslant \log 2), \\
C(T)=0(T>\log 2),
\end{gathered}
$$

and for $t \gg \dot{\gamma}^{-1}$ it is

$$
\begin{gathered}
C(T)=6 e^{-T / 2}+5 e^{-3 T / 2} / 2-3 e^{T / 2} \\
+e^{3 T / 2} / 2(0 \leqslant T \leqslant \log 2), \\
C(T)=3 e^{-3 T / 2} / 2(T>\log 2) .
\end{gathered}
$$

From the correlation function $C(T)$, we will calculate persistence exponents by employing two approximation methods. In one method [14], the "independent interval approximation" (IIA) is used. That is, the intervals between successive zeros of $\tilde{X}(T)$ are assumed to be statistically independent. We first define $A(T)=2 / \pi \sin ^{-1}[C(T)]$, which is equal to the correlation function of $\operatorname{sgn}[\tilde{X}(T)]$ for a Gaussian process. Then, we introduce the following function in terms of the Laplace transform of $A(T), \tilde{A}(s)$ :

$$
F(s)=1+(\langle T\rangle / 2) s[1-s \tilde{A}(s)],
$$

where $\langle T\rangle$ is the mean interval between successive zeros of $\tilde{X}(T)$, which equals $-2 / A /(0)$. $F(s)$ can be shown to have a simple zero at $s=-\theta$ under the IIA. For $t \gg \dot{\gamma}^{-1}$, the numerically obtained zero of $F(s)$ gives $\theta=0.67$, while for $t \ll \dot{\gamma}^{-1}$, we cannot apply this method because $\langle T\rangle=0$, which is readily confirmed from Eq. (9) and the definition of $\langle T\rangle$.

When $\langle T\rangle=0$, we can use another approximation method based on a variational expression for the persistence exponent [15]:

$$
\int_{0}^{+\infty} \frac{C(\tau) / C(0)-\exp (-\theta \tau)}{[1-\exp (-2 \theta \tau)]^{3 / 2}} d \tau=0 .
$$

The solution of this equation gives the persistence exponent $\theta$. This equation contains only the correlation function $C(T)$. By numerical calculations we get the two exponents $\theta=2.41$ $\left(t \ll \dot{\gamma}^{-1}\right)$ and $\theta=0.80\left(t \gg \dot{\gamma}^{-1}\right)$. For $t \gg \dot{\gamma}^{-1}$, this value is a little bit larger than the value of 0.67 obtained using the IIA. Comparing these exponents for $\tilde{x}(t)$ with that for $x(t)$, $1 / 2\left(t \ll \dot{\gamma}^{-1}\right)$ and $1 / 4\left(t \gg \dot{\gamma}^{-1}\right)$, we notice that the two

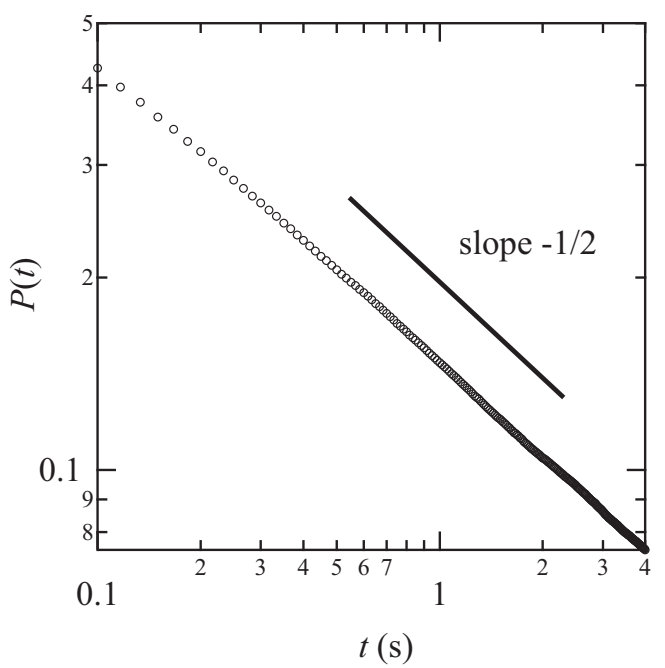

FIG. 3. Log-log plot of the persistence probability $P(t)$ of $x(t)$ for particles with a diameter of $0.5 \mu \mathrm{m}$ in distilled water at $25^{\circ} \mathrm{C}$. The solid line is a plot of $t^{-1 / 2}$. 

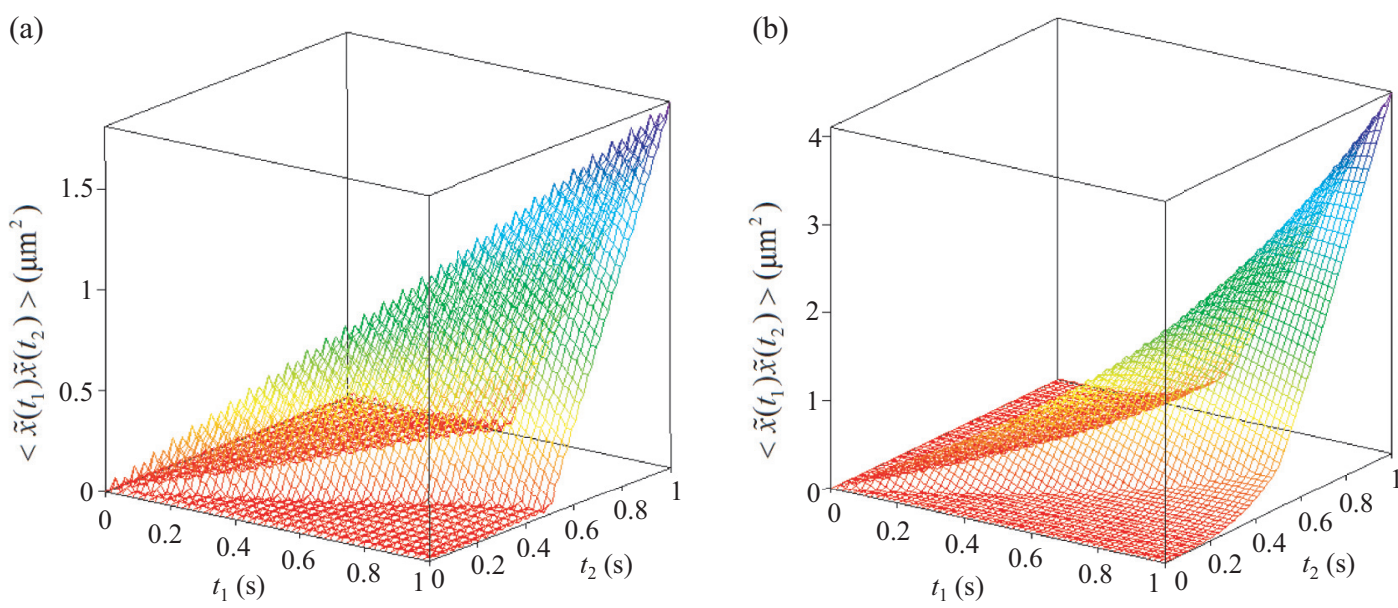

FIG. 4. (Color online) 3D plots of experimentally obtained two-time correlation functions of $\tilde{x}(t)$ for particles with a diameter of $0.5 \mu \mathrm{m}$ in distilled water at $25^{\circ} \mathrm{C}$ for (a) $\dot{\gamma}=0$ and (b) $\dot{\gamma}=2 \mathrm{~s}^{-1}$.

stochastic processes are different. There may be no simple relation between the persistence exponents for $\tilde{x}(t)$ and $x(t)$, but the stochastic nature of $\tilde{x}(t)$ depends on that of $x(t)$ so that the persistence exponent for $\tilde{x}(t)$ does reflect the stochastic nature of $x(t)$, as is seen in the shear rate dependence of the persistence exponent.

\section{EXPERIMENTAL RESULTS AND DISCUSSION}

For observations of particles under a shear flow, we used a system combining a cone-plate rheometer and a confocal scanning laser microscope (CSLM), as shown in Fig. 2. Observations of fluorescent beads dispersed in distilled water were made through the bottom glass plate of the rheometer with the CSLM [Fig. 2(a)]. The observations were taken $4 \mathrm{~mm}$ inward from the edge of the rotating plate, where the gap was $170 \mu \mathrm{m}$. The focal plane of the CSLM was fixed at $10 \mu \mathrm{m}$ above the glass surface and two-dimensional images $(400 \mu \mathrm{m} \times 400 \mu \mathrm{m})$ were captured with a frame rate of $60 \mathrm{fps}$ for about $2 \mathrm{~min}$. We used a low numerical aperture (NA) object lens $(20 \times$ with $\mathrm{NA}=0.50)$ so that we could observe particles in a layer with a thickness of about $10 \mu \mathrm{m}$ between $z_{1}=5 \mu \mathrm{m}$ and $z_{2}=15 \mu \mathrm{m}$ [Fig. 2(b)] by adjusting the value used for image thresholding. Under our experimental conditions, the diffusion length along the $z$ axis is smaller than $z_{1}$ so that boundary effects can be neglected. All the particles in this layer were tracked and the time dependences of the $x$ and $y$ coordinates were obtained. In the measurements, fluorescent polystyrene spheres with a diameter of $0.5 \mu \mathrm{m}$ (FluoSpheres, Invitrogen) were used, dispersed dilutely in distilled water so that interparticle interaction was negligible. Measurements were made at shear rates of $0,1,2$, and $4 \mathrm{~s}^{-1}$ at $25^{\circ} \mathrm{C}$.

Let us first examine the persistence probability $P(t)$ of $x(t)$ for free Brownian motion at $\dot{\gamma}=0$, the exponent of which is expected to be $1 / 2$. We used about 700 traces of different particles with durations greater than $2.5 \mathrm{~s}$ for one run to calculate the persistence probability. These were then averaged over four runs to reduce statistical errors. The double logarithmic plot of the persistence probability is shown in Fig. 3, in which the solid line indicates a slope of $-1 / 2$. It can be seen that the persistence probability decays as a power law with the theoretically expected exponent.

Three-dimensional plots of the two-time correlation function of $\tilde{x}(t)$ for $\dot{\gamma}=0$ and $2 \mathrm{~s}^{-1}$ are shown in Figs. 4(a) and 4(b), respectively. For $\dot{\gamma}=0$, the values in the regions $t_{1}>2 t_{2}$ and $t_{2}>2 t_{1}$ are almost zero, and the $\operatorname{MSD}\left(t=t_{1}=t_{2}\right)$ is proportional to $t$. These features agree with the theoretical results [Fig. 1(a)]. On the other hand, upon application of a shear flow [Fig. 4(b)], long correlation appears in the regions $t_{1}>2 t_{2}$ and $t_{2}>2 t_{1}$, and the contribution from the $t^{3}$ term appears in the $\operatorname{MSD}\left(t=t_{1}=t_{2}\right)$. These results are quantitatively in good agreement with the theoretical ones [Fig. 1(b)].

Finally, we show the persistence probabilities of $\tilde{x}(t)$ for $\dot{\gamma}=0,1,2$, and $4 \mathrm{~s}^{-1}$ in Fig. 5, in which the theoretically obtained slopes (exponents) are also depicted. Without a shear

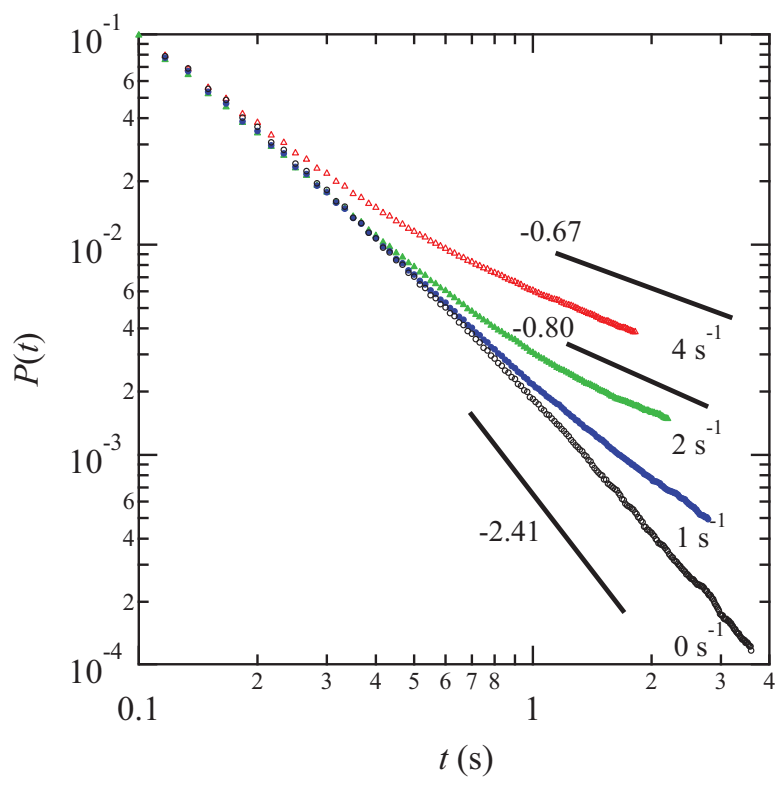

FIG. 5. (Color online) Log-log plot of the persistence probability $P(t)$ of $\tilde{x}(t)$ for particles with a diameter of $0.5 \mu \mathrm{m}$ in distilled water at $25^{\circ} \mathrm{C}$ for $\dot{\gamma}=0,1,2$, and $4 \mathrm{~s}^{-1}$. 
flow at $\dot{\gamma}=0$, the persistence exponent is very close to the theoretical value, 2.41 . Note that this value is totally different from the value of $1 / 2$ for $x(t)$. In the presence of a shear flow, on the other hand, the long-time behavior changes. For $\dot{\gamma}=1 \mathrm{~s}^{-1}$, the slope gradually increases with time, but it seems that the slope does not reach a constant value. It is difficult in our experiment to obtain the persistence probability at longer times. For $\dot{\gamma}=2 \mathrm{~s}^{-1}$, however, we can see a constant slope at long times. This slope is close to the theoretical results of -0.67 (IIA) or -0.8 (variational method). However, it is difficult to judge from our experiments which is the better approximation. For $\dot{\gamma}=4 \mathrm{~s}^{-1}$, the slope is almost the same as that for $\dot{\gamma}=2 \mathrm{~s}^{-1}$.

\section{CONCLUSIONS}

We have investigated the two-time correlation function and persistence probability for sheared Brownian particles by using a confocal laser microscope. Without a shear flow (i.e., at equilibrium) it was clearly confirmed that the persistence exponent of $x(t)$ is $1 / 2$, as predicted theoretically. To analyze the data under a shear flow we used $\tilde{x}(t)$, which eliminates the effects of convection, instead of $x(t)$. We calculated the two-time correlation function and persistence exponent of $\tilde{x}(t)$, and obtained two time regimes with different exponents: $\theta=2.41$ (IIA) for $t \ll \dot{\gamma}^{-1}$ and $\theta=0.67$ (IIA) or $\theta=0.80$ (variational method) for $t \gg \dot{\gamma}^{-1}$. These two regimes correspond to those predicted by Chakraborty [12], though the values of persistence exponents are different. The theoretical results were compared with experimental observations, and good agreement was obtained both in the two regimes.

\section{ACKNOWLEDGMENT}

This work was supported by a Grant-in-Aid for Scientific Research on Innovative Areas "Fluctuation \& Structure" (No. 25103006) and JSPS Fellows (Contract No. 25-2447) from the Ministry of Education, Culture, Sports, Science and Technology of Japan.
[1] J. Krug, H. Kallabis, S. N. Majumdar, S. J. Cornell, A. J. Bray, and C. Sire, Phys. Rev. E 56, 2702 (1997).

[2] Z. Toroczkai, T. J. Newman, and S. Das Sarma, Phys. Rev. E 60, R1115 (1999).

[3] M. Constantin, C. Dasgupta, P. Punyindu Chatraphorn, S. N. Majumdar, and S. Das Sarma, Phys. Rev. E 69, 061608 (2004).

[4] S. N. Majumdar, A. J. Bray, S. J. Cornell, and C. Sire, Phys. Rev. Lett. 77, 3704 (1996).

[5] M. R. Swift and A. J. Bray, Phys. Rev. E 59, R4721 (1999).

[6] T. W. Burkhardt, J. Phys. A 33, L429 (2000).

[7] D. S. Fisher, P. Le Doussal, and C. Monthus, Phys. Rev. Lett. 80, 3539 (1998).
[8] P. Le Doussal, C. Monthus, and D. S. Fisher, Phys. Rev. E 59, 4795 (1999).

[9] D. Chakraborty, Eur. Phys. J. B 64, 263 (2008).

[10] S. Bhattacharya, D. Das, and S. N. Majumdar, Phys. Rev. E 75, 061122 (2007).

[11] D. Chakraborty, Phys. Rev. E 85, 051101 (2012).

[12] D. Chakraborty, Eur. Phys. J. B 85, 281 (2012).

[13] S. N. Majumdar, Curr. Sci. 77, 370 (1999).

[14] S. N. Majumdar, C. Sire, A. J. Bray, and S. J. Cornell, Phys. Rev. Lett. 77, 2867 (1996).

[15] C. Sire, S. N. Majumdar, and A. Rudinger, Phys. Rev. E 61, 1258 (2000).

[16] H. Orihara and Y. Takikawa, Phys. Rev. E 84, 061120 (2011). 